\title{
NGAL for the detection of acute kidney injury in the emergency room
}

\begin{abstract}
"...clinical trials of acute kidney injury therapeutics should utilize biomarkers such as NGAL as secondary outcome measures that can provide sensitive real-time evidence for efficacy that may be missed by delayed functional markers such as serum creatinine alone."
\end{abstract}

\section{KEYWORDS: acute kidney injury $\approx$ acute renal failure $\approx$ biomarker $\approx$ emergency setting - neutrophil gelatinase-associated lipocalin}

\section{Acute kidney injury in the emergency room}

The incidence of acute kidney injury (AKI) is increasing globally [1]. This condition accounts for $3-5 \%$ of admissions in general hospitals, many of whom initially present to emergency room (ER) settings with community-acquired kidney damage from diverse etiologies. In contrast to hospital-acquired AKI, where the occurrence and timing of AKI may be predictable based on clinical scoring systems, the diagnosis of AKI in the ER setting is fraught with difficulty. First, community-acquired AKI often strikes previously healthy individuals with no known risk factors. Second, the heterogeneous insults leading to AKI are often encountered well before presentation to the ER. Third, a single measurement of serum creatinine, the current standard marker of kidney function, cannot distinguish true structural (intrinsic) AKI from chronic kidney disease (CKD) or from functional volume-responsive AKI (prerenal azotemia). It is critical to make these distinctions in the ER, since the medical management of each is dramatically different and mismanagement is deleterious. Fourth, creatinine levels are influenced by several nonrenal factors such as age, gender, muscle mass, muscle metabolism, medication use, hydration status and nutrition status. Biomarkers to improve detection of early AKI and provision of early interventions are therefore a subject of intense contemporary interest [2].

The discovery of novel AKI biomarkers was accelerated by the utilization of unbiased functional genomic platforms that identified NGAL as the most upregulated gene in the kidney very early after AKI in multiple animal models [3]. NGAL protein expression was detected in the injured kidneys in tubule epithelial cells undergoing proliferation and regeneration, indicating a critical role in the repair process $[4,5]$. The serendipitous detection of NGAL in the urine and plasma in animal and human AKI has resulted in rigorous studies that have established the biological plausibility of NGAL as an early AKI biomarker [6]. We now know that urine NGAL originates specifically from damaged epithelial cells of the distal nephron, whereas plasma NGAL is derived from both the injured kidneys (via tubular backleak) and from extrarenal organs that the kidney crosstalks with.

These robust preclinical studies spawned a number of translational research efforts in humans. Landmark studies in settings such as cardiac surgery $[7,8]$ established the many desirable characteristics of NGAL as a biomarker to predict hospital-acquired AKI and its adverse consequences. Attention has now been turned to NGAL as a biomarker of AKI in the heterogeneous ER setting, with studies that are summarized below.

\section{NGAL in the emergency room}

Nickolas and colleagues reported the first study of urine NGAL in the ER [9]. In this single-center prospective study, 635 patients admitted to the hospital from the ER were subsequently adjudicated to one of four groups using established diagnostic criteria: intrinsic AKI, prerenal azotemia, nonprogressive CKD and normal kidney function. A single measurement of NGAL (by immunoblotting) in urine obtained at initial presentation distinguished intrinsic AKI from the other clinical groups. An elevated initial urinary NGAL level was an outstanding predictor of intrinsic AKI, with an area under the receiver operating characteristic curve (AUC) of 0.95 , sensitivity of $99 \%$ and specificity approaching $100 \%$.

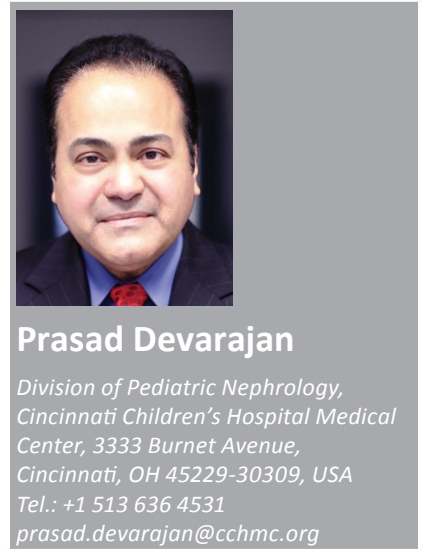

Muture $\mathrm{fSg}$ 
Furthermore, an elevated urinary NGAL level was highly predictive of adverse clinical outcomes, including need for nephrology consultation, dialysis and admission to the intensive care unit (odds ratio [OR]: 24.71; CI: 7.69-79.42). These findings have now been confirmed in a large, multicenter prospective cohort of 1635 unselected ER patients who were subsequently hospitalized [10]. At a cutoff concentration of $104 \mathrm{ng} / \mathrm{ml}$, the admission urinary NGAL predicted intrinsic AKI (AUC: 0.81 ; specificity: $81 \%$ ), the severity and duration of intrinsic AKI, and a composite outcome of dialysis requirement or death. Analysis of the net reclassification improvement and the integrated discrimination improvement revealed a significant improvement in predictive ability when urinary NGAL was added to clinical risk-prediction models. Another strength of this study was the use of a standardized clinical platform for urine NGAL measurement (ARCHITECT $^{\circledR}$, Abbott Laboratories, IL, USA), which provides accurate measurements (coefficients of variation $<3 \%$ ) and a rapid turn-around time of approximately $30 \mathrm{~min}$.

\section{"...early measurements of NGAL in the urine or plasma are highly predictive of acute kidney injury..."}

Shapiro and colleagues studied the diagnostic accuracy of plasma NGAL for the prediction of $A K I$ in the ER [11]. In this 661-patient multicenter cohort study, a plasma NGAL level of greater than $150 \mathrm{ng} / \mathrm{ml}$ measured at the time of ER presentation predicted AKI occurring within the first 3 days of hospitalization, with a sensitivity of $96 \%$ and an AUC of 0.82. This study employed a rapid point-of-care platform for plasma NGAL measurement (Triage ${ }^{\circledR}$, Biosite Incorporated, CA, USA), which provides results within $15 \mathrm{~min}$ and with clinical grade accuracy. Independent validation of these results derive from a recent prospective study by Soto and colleagues of 616 patients admitted for subsequent clinical adjudication, who had plasma NGAL levels measured in the ER using the Triage assay [12]. Elevated plasma NGAL values obtained within $12 \mathrm{~h}$ of hospitalization reliably distinguished intrinsic AKI from prerenal azotemia and CKD (AUC: 0.82), and predicted the severity of AKI. The addition of plasma NGAL to the clinical model yielded an impressive net classification improvement of $94.3 \%$ and an integrated discrimination improvement of 0.122 . The authors constructed a three-zone partition of NGAL levels based on generalized additive models. A plasma NGAL value $<97 \mathrm{ng} / \mathrm{ml}$ predicted a low risk for AKI, and a value $>133 \mathrm{ng} / \mathrm{ml}$ portended a very high risk (OR: 9.8; CI: 5.6-16.9). Patients in the 'gray zone' with initial plasma NGAL values between 97 and $133 \mathrm{ng} / \mathrm{ml}$ were at intermediate risk for AKI (OR: 4.6; CI: 2.3-8.6).

Collectively, the published literature on NGAL as a biomarker in the ER setting reveals that it is:

- Easily, rapidly and reliably measureable at the bedside with a standardized point-of-care platform or in the laboratory with a standard clinical platform;

- Noninvasive, using easily accessible samples such as blood or urine;

- Increased proportional to the degree and severity of AKI;

- Sensitive enough to facilitate early detection and accurate prediction, with a wide dynamic range and cutoff values that allow for risk stratification;

- Specific to intrinsic AKI, and clearly discerns AKI from pre-renal azotemia and CKD;

- Of added value to baseline clinical risk assessment of AKI;

- Associated with a known pathophysiologic mechanism.

\section{Limitations \& future directions}

A critical review of published studies that have examined more than 3500 subjects in the ER setting indicates that early measurements of NGAL in the urine or plasma are highly predictive of AKI, with an excellent overall AUC of 0.85 [9-12]. However, the following limitations should be acknowledged:

- Urine NGAL values may be confounded by hydration status and urine output, and may therefore need standardization by expressing as a ratio with urine creatinine;

- Plasma NGAL values may be confounded by systemic infections, which can elevate NGAL levels (although generally not to the same extent as in patients with AKI);

- While patients with stable CKD display low levels of NGAL, those with progressive CKD may exhibit higher concentrations that may approach levels measured in mild AKI;

- The type of assay used matters, since it is the monomeric form of NGAL that is secreted by the acutely injured kidney, whereas the dimer is the predominant form secreted by neutrophils, and CKD is characterized by the 
appearance of multiple NGAL forms; fortunately, the standardized clinical platforms predominantly detect the monomeric forms [13];

- Specific cutoff values depend on the type of sample, the clinical setting and the assay platform;

- The literature is based on NGAL measurements in stored samples, with confounding effects of prolonged storage, degradation and effect of freeze-thaw cycles;

- The literature has included a myriad of AKI definitions, all based on an increase in serum creatinine, which is at best a flawed bronze standard that underestimates the diagnostic accuracy of NGAL for predicting true intrinsic AKI.

Even patients with 'subclinical' AKI, who do not satisfy serum creatinine-based AKI diagnosis but have NGAL levels above the described cutoffs, display a threefold increased risk of mortality or need for renal replacement therapy [14].

\section{"In the clinical setting, biomarker measurements should be reserved for individuals at clinical risk for acute kidney injury, and the biomarker should continue to demonstrate added value beyond clinical risk scores."}

It is recommended that future research studies employ a multicenter approach, with pertinent clinical end points (and not merely serum creatinine-based definitions) to further establish the diagnostic and prognostic value of NGAL as an AKI biomarker. In the clinical setting, biomarker measurements should be reserved for individuals at clinical risk for AKI, and the biomarker should continue to demonstrate added value beyond clinical risk scores. For both clinical and research purposes, the use of freshly collected serial urine or blood specimens and measurement of NGAL on established clinical laboratory platforms is recommended for maximal translatability to clinical practice. The ultimate adoption of NGAL will depend on successful interventional trials that utilize early biomarker measurements for patient randomization with a resultant improvement in patient outcomes. It is also recommended that clinical trials of AKI therapeutics should utilize biomarkers such as NGAL as secondary outcome measures that can provide sensitive real-time evidence for efficacy that may be missed by delayed functional markers such as serum creatinine alone.

\section{Financial \& competing interests disclosure \\ $P$ Devarajan is supported by a grant from the NIH (P50 DK096418), and is a coinventor on submitted patents on NGAL as a marker of kidney injury. The author has no other relevant affiliations or financial involvement with any organization or entity with a financial interest in or financial conflict with the subject matter or materials discussed in the manuscript apart from those disclosed. \\ No writing assistance was utilized in the production of this manuscript.}

\section{References}

1 Lameire NH, Bagga A, Cruz D et al. Acute kidney injury: an increasing global concern. Lancet 382(9887), 170-179 (2013).

2 Devarajan P. Neutrophil gelatinaseassociated lipocalin: a promising biomarker for human acute kidney injury. Biomarkers Med. 4(2), 265-280 (2010)

3 Supavekin S, Zhang W, Kucherlapati R et al. Differential gene expression following early renal ischemia/reperfusion. Kidney Int. 63(5), 1714-1724 (2003).

4 Mishra J, Ma Q, Prada A et al. Identification of neutrophil gelatinase-associated lipocalin as a novel early urinary biomarker for ischemic renal injury. J. Am. Soc. Nephrol. 14(10), 2534-2543 (2003).

5 Mishra J, Mori K, Ma Q et al. Amelioration of ischemic acute renal injury by neutrophil gelatinase-associated lipocalin. J. Am. Soc. Nephrol. 15(12), 3073-3082 (2004).
6 Paragas N, Qiu A, Zhang Q et al. The NGAL reporter mouse detects the response of the kidney to injury in real time. Nat. Med. 17(2), 216-222 (2011).

7 Mishra J, Dent C, Tarabishi R et al. Neutrophil gelatinase-associated lipocalin (NGAL) as a biomarker for acute renal injury after cardiac surgery. Lancet 365(9466), 1231-1238 (2005).

8 Bennett M, Dent CL, Ma Q et al. Urine NGAL predicts severity of acute kidney injury after cardiac surgery: a prospective study. Clin. J. Am. Soc. Nephrol. 3(3), 665-673 (2008).

9 Nickolas TL, O'Rourke MJ, Yang J et al. Sensitivity and specificity of a single emergency department measurement of urinary neutrophil gelatinase-associated lipocalin for diagnosing acute kidney injury. Ann. Intern. Med. 148(11), 810-819 (2008).

10 Nickolas TL, Schmitt-Ott KM, Canetta P et al. Diagnostic and prognostic stratification in the emergency department using urinary biomarkers of nephron damage. J. Am. Coll. Cardiol. 59(3), 246-255 (2012).

11 Shapiro NI, Trzeciak S, Hollander JE et al. The diagnostic accuracy of plasma neutrophil gelatinase-associated lipocalin in the prediction of acute kidney injury in emergency department patients with suspected sepsis. Ann. Emerg. Med. 56(1), 52-59 (2010).

12 Soto K, Papoila AL, Coelho S et al. Plasma NGAL for the diagnosis of AKI in patients admitted from the emergency department setting. Clin. J. Am. Soc. Nephrol. 8(12), 2053-2063 (2013).

13 Nickolas TL, Forster C, Sise ME et al. NGAL $(\mathrm{Lcn} 2)$ monomer is associated with tubulointerstitial damage in chronic kidney disease. Kidney Int. 82(6), 718-722 (2012).

14 Haase M, Devarajan P, Haase-Fielitz A et al. The outcome of neutrophil gelatinaseassociated lipocalin-positive subclinical acute kidney injury: a multicenter pooled analysis of prospective studies. J. Am. Coll. Cardiol. 57(17), 1752-1761 (2011). 